Journal of Mathematics and Statistics 6 (1): 60-63, 2010

ISSN 1549-3644

(C) 2010 Science Publications

\title{
A Multiderivative Collocation Method for 5th Order Ordinary Differential Equations
}

\author{
S.J. Kayode and D.O. Awoyemi \\ Department of Mathematical Sciences, School of Sciences, \\ Federal University of Technology, Postal Code: +234, PMB 704, Akure, Ondo State, Nigeria
}

\begin{abstract}
Problem statement: The conventional methods of solving higher order differential equations have been by reducing them to systems of first order equations. This approach is cumbersome and increases computational time. Approach: To address this problem, a numerical algorithm for direct solution of 5th order initial value problems in ordinary differential equations (odes), using power series as basis function, is proposed in this research. Collocation of the differential system is taken at selected grid points to reduce the number of functions to be evaluated per iteration. A number of predictors and their derivatives having the same order of accuracy with the main method are proposed. Results: The approach yields a multiderivative method of order six. Numerical examples solved show increased efficiency of the method with increased number of iterations, converging to the theoretical solutions. Conclusion/Recommendations: The new mutiderivative method is efficient to solve linear and nonlinear fifth order odes without reduction to system of lower order equations.
\end{abstract}

Key words: Predictors, interval of absolute stability, error constant, differential system

\section{INTRODUCTION}

In this research, numerical method of solution of higher order differential equations of the form:

$$
\begin{array}{r}
y^{(m)}=f\left(x, y, y^{\prime}, \ldots, y^{m-1}\right), y(a)=y_{0}, \\
y^{i}(a)=y_{i}, i=1(1) m-1, m \geq 2
\end{array}
$$

is considered for step number $\mathrm{k} \geq 5$. This class of Problems (1) has a lot of applications in Science and Engineering, especially in mechanical systems, control theory and celestial mechanics.

The practice of solving this type of problems has been the reduction to systems of first-order equations and the resulting equations solved by applying any suitable method for first order equations (Awoyemi, 2003). In the same article, it is extensively discussed that due to the dimension of the problem after it has been reduced to a system of first order equations, the approach waste a lot of human efforts and computer time.

Eminent scholars have contributed significantly in their works in this area of research to solving Problem (1) using different numerical methods (Lambert, 1973; Jacques and Judd, 1987; Adee et al., 2005; Awoyemi, 2005; Kayode and Awoyemi, 2005; Awoyemi and Idowu, 2005; Fatunla, 1988; 1994; Kayode, 2008a).
Attempts have been made by some researchers to solve directly Problem (1) for $\mathrm{m}=4$ by developing methods of step number $\mathrm{k}=4$ with varying order of accuracy (Awoyemi, 2005; Kayode, 2008b). But none of these could handle Problem (1) directly when $m>4$ without reducing it to a system of lower order problems.

In this article, Problem (1) is solved directly by developing a 5 step multiderivative method for $m=5$.

\section{MATERIALS AND METHODS}

A power series of a single variable $\mathrm{x}$ in the form:

$$
P(x)=\sum_{j=0}^{\infty} a_{j} x^{j}
$$

is used as trial function to produce the approximate solution as:

$$
\begin{aligned}
& y(x)=\sum_{j=0}^{k+2} \lambda_{j} x^{j} \\
& \lambda_{j} \in R, j=0(1) k+1, x \in[a, b], y \in C^{m}(a, b) \subset P(x) .
\end{aligned}
$$

The 5 th derivative of (3) is:

Corresponding Author: S.J. Kayode, Department of Mathematical Sciences, School of Sciences, Federal University of Technology, Postal Code: +234, PMB 704, Akure, Ondo State, Nigeria Tel: +2348033974438 
$y^{v}(x)=\sum_{j=0}^{k+2} j(j-1)(j-2)(j-3)(j-4) \lambda_{J} x^{j-5}$

Thus from Eq. 1 and 4 we have:

$\sum_{j=0}^{k+2} j(j-1)(j-2)(j-3)(j-4) \lambda_{j} x^{j-5}=f\left(x, y, y^{\prime}, y^{\prime \prime}, y^{\prime \prime \prime}, y^{1 v}\right)$

Collocating Eq. 5 at $\mathrm{x}=\mathrm{x}_{\mathrm{n}+2 \mathrm{j}+1}, \mathrm{j}=0(1) 2$ and interpolating (3) at $\mathrm{x}=\mathrm{x}_{\mathrm{n}+\mathrm{j}}, \mathrm{j}=0(1) 4$ yield the following systems of equations:

$\sum_{j=0}^{k+2} j(j-1)(j-1)(j-2)(j-3)(j-4) \lambda_{j} x_{n+i}^{j-5}=f_{n+2 i+1}$,

$\mathrm{i}=0(1) 2$

$\sum_{j=0}^{k+2} \lambda_{j} x_{n+j}=y_{n+j}, j=0(1) 4$

After solving the system of Eq. 7 and 8 for the values of $\lambda_{\mathrm{j}}$ 's and substituting these values into Eq. 3, with the necessary algebraic manipulations, our method is obtained to be:

$y_{5}(x)=\sum_{j=0}^{4} \alpha_{j}(x) y_{n+j}+\sum_{j=0}^{2} \beta_{2 j+1}(x) f_{n+2 j+1}$

From (8) the coefficients $\alpha_{\mathrm{j}}(\mathrm{x})$ and $\beta_{\mathrm{j}}(\mathrm{x})$ are given as follows:

$$
\begin{aligned}
& \alpha_{0}(t)=\frac{1}{24}\left[t^{4}+6 t^{3}+11 t^{2}+6 t\right] \\
& \alpha_{1}(t)=-\frac{1}{6}\left[t^{4}+7 t^{3}+14 t^{2}+8 t\right] \\
& \alpha_{2}(t)=\frac{1}{4}\left[t^{4}+8 t^{3}+19 t^{2}+12 t\right] \\
& \alpha_{3}(t)=-\frac{1}{6}\left[t^{4}+9 t^{3}+26 t^{2}+24 t\right] \\
& \alpha_{4}(t)=\frac{1}{24}\left[t^{4}+10 t^{3}+35 t^{2}+50 t+24\right] \\
& \beta_{5}(t)=\frac{h^{5}}{20160}\left[\begin{array}{l}
t^{7}+14 t^{6}+63 t^{5}+70 t^{4}- \\
196 t^{3}-504 t^{2}-288 t
\end{array}\right] \\
& \beta_{3}(t)=\frac{h^{5}}{10080}\left[\begin{array}{l}
-t^{7}-7 t^{6}+63 t^{5}+735 t^{4}+ \\
2506 t^{3}+3472 t^{2}+1632 t
\end{array}\right] \\
& \beta_{1}(t)=\frac{h^{5}}{20160}\left[\begin{array}{l}
t^{7}-21 t^{5}+140 t^{4}+1064 t^{3}+ \\
1960 t^{2}+1056 t
\end{array}\right]
\end{aligned}
$$

The discrete scheme and its first, second, third and fourth derivatives arising from Eq. 9 are as put below when $t=1$, which implies that $x=x_{n+4}$ :

$$
\begin{aligned}
& \mathrm{y}_{\mathrm{n}+5}-5 \mathrm{y}_{\mathrm{n}+4}+10 \mathrm{y}_{\mathrm{n}+3}-10 \mathrm{y}_{\mathrm{n}+2}+5 \mathrm{y}_{\mathrm{n}+1}-\mathrm{y}_{\mathrm{n}}= \\
& \frac{\mathrm{h}^{5}}{24}\left[-\mathrm{f}_{\mathrm{n}+6}+20 \mathrm{f}_{\mathrm{n}+3}+5 \mathrm{f}_{\mathrm{n}+1}\right]
\end{aligned}
$$

Order $\mathrm{p}=6$, error constant $\mathrm{c}_{\mathrm{p}+2}=0.2083$ and interval of absolute stability is $\mathrm{X}(\theta)=(0,32)$ :

$$
\begin{aligned}
y_{n+5}^{\prime}= & \frac{1}{12 h}\left[\begin{array}{l}
77 y_{n+4}-214 y_{n+3}+ \\
234 y_{n++2}-122 y_{n+1}+25 y_{n}
\end{array}\right] \\
& +\frac{h^{4}}{10080}\left[-599 f_{n+5}+19360 f_{n+3} 4315 f_{n+1}\right]
\end{aligned}
$$

with:

$$
\mathrm{p}=6, \mathrm{c}_{\mathrm{p}+2}=-0.400297619
$$

$$
\begin{aligned}
& \mathrm{y}_{\mathrm{n}+5}^{\prime \prime}=\frac{1}{12 \mathrm{~h}^{2}}\left[\begin{array}{l}
71 \mathrm{y}_{\mathrm{n}+4}-236 \mathrm{y}_{\mathrm{n}+3}+ \\
294 \mathrm{y}_{\mathrm{n}+2}-164 \mathrm{y}_{\mathrm{n}+1}+35 \mathrm{y}_{\mathrm{n}}
\end{array}\right] \\
& +\frac{\mathrm{h}^{3}}{1440}\left[27 \mathrm{f}_{\mathrm{n}+5}+4544 \mathrm{f}_{\mathrm{n}+3}+829 \mathrm{f}_{\mathrm{n}+1}\right] \\
& \mathrm{p}=6, \mathrm{c}_{\mathrm{p}+2}=-0.442890211 \\
& \mathrm{y}_{\mathrm{n}+5}^{\prime \prime \prime}=\frac{1}{2 \mathrm{~h}^{3}}\left[7 \mathrm{y}_{\mathrm{n}+4}-26 \mathrm{y}_{\mathrm{n}+3}+36 \mathrm{y}_{\mathrm{n}+2}-22 \mathrm{y}_{\mathrm{n}+2}+5 \mathrm{y}_{\mathrm{n}}\right] \\
& +\frac{\mathrm{h}^{2}}{160}\left[49 \mathrm{f}_{\mathrm{n}+5}+562 \mathrm{f}_{\mathrm{n}+3}+69 \mathrm{f}_{\mathrm{n}+1}\right] \\
& \mathrm{p}=6 \mathrm{c}_{\mathrm{p}+2}=-0.09583333 . \\
& \mathrm{y}_{\mathrm{n}+5}^{\text {iv }}=\frac{1}{\mathrm{~h}^{4}}\left[\mathrm{y}_{\mathrm{n}+4}-4 \mathrm{y}_{\mathrm{n}+3}+6_{\mathrm{n}+2}-4 \mathrm{y}_{\mathrm{n}+1}+\mathrm{y}_{\mathrm{n}}\right] \\
& +\frac{\mathrm{h}}{12}\left[9 \mathrm{f}_{\mathrm{n}+5}+26 \mathrm{f}_{\mathrm{n}+3}+\mathrm{f}_{\mathrm{n}+1}\right]
\end{aligned}
$$

$\mathrm{p}=6, \mathrm{c}_{\mathrm{p}+2}=0.35972222$

Predictors: Except that collocation is not taken at $\mathrm{x}=\mathrm{x}_{\mathrm{n}+5}$, the procedure for the development of the main predictor to calculate $\mathrm{y}_{\mathrm{n}+5}$ and its required derivatives is the same as for the main method. For $y_{n+j}, j=1(1) 4$, the predictors are derived by Taylor series method. The discrete schemes and their required derivatives arising from the predictors are as listed below: 
J. Math. \& Stat., 6 (1): 60-63, 2010

$\mathrm{y}_{\mathrm{n}+5}=5 \mathrm{y}_{\mathrm{n}+6}-10 \mathrm{y}_{\mathrm{n}+5}+10 \mathrm{y}_{\mathrm{n}+4}-5 \mathrm{y}_{\mathrm{n}+3}+\mathrm{y}_{\mathrm{n}}$

$+\frac{h^{5}}{24}\left[5 f_{n+4}+20 f_{n+2}-f_{n}\right]$

$p=6$, error constant $c_{p+2}=-0.2083333$, interval of absolute stability $\mathrm{X}(\theta)=(-31.94,0)$ :

$$
\begin{aligned}
y_{n+5}^{\prime}= & \frac{1}{12 h}\left[\begin{array}{l}
77 y_{n+4}-214 y_{n+3}+ \\
234 y_{n+2}-122 y_{n+1}+25 y_{n}
\end{array}\right] \\
& +\frac{h^{4}}{10080}\left[5575 f_{n+4}+18460 f_{n+2}-1014 f_{n}\right]
\end{aligned}
$$

$\mathrm{p}=6$, error constant $\mathrm{c}_{\mathrm{p}+2}=0.49156746$ :

$$
\begin{aligned}
y_{n+5}^{\prime \prime}= & \frac{1}{12 h^{2}}\left[\begin{array}{l}
71 y_{n+4}-236 y_{n+3}+ \\
294 y_{n+2}-164 y_{n+1}+35 y_{n}
\end{array}\right] \\
& +\frac{h^{3}}{1440}\left[1651 f_{n+4}+3996 f_{n+2}-247 f_{n}\right]
\end{aligned}
$$

$\mathrm{p}=6$, error constant $\mathrm{c}_{\mathrm{p}+2}=0.800165343$ :

$$
\begin{aligned}
y_{n+5}^{\prime \prime \prime}= & \frac{1}{2 h^{3}}\left[7 y_{n+4}-26 y_{n+3}+36 y_{n+2}-22 y_{n+1}+5 y_{n}\right] \\
& +\frac{h^{2}}{80}\left[147 f_{n+4}+206 f_{n+2}-13 f_{n}\right]
\end{aligned}
$$

Order $\mathrm{p}=6$, error constant $\mathrm{c}_{\mathrm{p}+2}=0.69716666$ :

$$
\begin{aligned}
y^{i v}{ }_{n+5}= & \frac{1}{h^{4}}\left[y_{n+4}-4 y_{n+3}+6 y_{n+2}-4 y_{n+1}+y_{n}\right] \\
& +\frac{h}{24}\left[53 f_{n+4}+18 f_{n+2}+f_{n}\right]
\end{aligned}
$$

$\mathrm{p}=6$, error constant $\mathrm{c}_{\mathrm{p}+2}=-0.47361111$.

Finally $y_{n+j}=j 1(1) 4$ and their first, second, third and fourth derivatives are determined by Taylor's method as follows:

$$
\begin{aligned}
& y_{n+i}=y\left(x_{n}+i h\right)=y\left(x_{n}\right)+\sum_{j=1}^{7} \frac{(i h)^{j} y^{j}\left(x_{n}\right)}{j I}, \quad i=1(1) 4 \\
& y_{n+i}^{i}=y^{i}\left(x_{n}+i h\right)=y^{i}\left(x_{n}\right)+\sum_{j=1}^{7} \frac{(i h)^{j} y^{j+1}\left(x_{n}\right)}{j I}, i=1(1) 4 \\
& f_{n}=f\left(x_{n}, y_{n}, y_{n}^{\prime}, y_{n}^{\prime \prime}, y_{n}^{\prime \prime \prime}, y^{1 v}{ }_{n}\right) \\
& f_{n}^{\prime}=f^{\prime}\left(x_{n}, y_{n}, y_{n}^{\prime}, y_{n}^{\prime \prime}, y_{n}^{\prime \prime \prime}, y^{1 v}{ }_{n}\right)
\end{aligned}
$$

$$
f_{n}^{\prime \prime}=f^{\prime \prime}\left(x_{n}, y_{n}, y_{n}^{\prime}, y_{n}^{\prime \prime}, y_{n}^{\prime \prime \prime}, y^{1 v}{ }_{n}\right)
$$

Furthermore, $f_{n}^{\prime}$ and $f_{n}^{\prime \prime}$ are expressed in partial derivatives as follows:

$$
\begin{aligned}
f^{\prime} & =\frac{d f}{d x}=\frac{\partial f}{\partial x}+y^{\prime} \frac{\partial f}{\partial y}+y^{\prime \prime} \frac{\partial f}{\partial y^{\prime}}+y^{\prime \prime \prime} \frac{\partial f}{\partial y^{\prime \prime}}+y^{1 v} \frac{\partial f}{\partial y^{\prime \prime \prime}}+f \frac{\partial f}{\partial y^{1 v}}, y^{i} \\
& =\frac{d^{i} y}{d x^{i}}, i=1,2,3,4 \ldots \\
f^{\prime \prime} & =\frac{d^{2} f}{d x^{2}}=2\left(A y^{\prime}+B y^{\prime \prime}+C y^{\prime \prime \prime}+D f^{1 v}+E f\right)+F+G+H
\end{aligned}
$$

Where:

$$
\begin{aligned}
& A=\frac{\partial^{2} \mathrm{f}}{\partial x \partial y}+y^{\prime \prime} \frac{\partial^{2} \mathrm{f}}{\partial y_{\partial y^{\prime}}}+y^{\prime \prime \prime} \frac{\partial^{2} f}{\partial y \partial y^{\prime \prime}}+y^{1 v} \frac{\partial^{2} f}{\partial y \partial y^{\prime \prime \prime}}+f \frac{\partial^{2} f}{\partial y \partial y^{1 v}} \\
& B=\frac{\partial^{2} f}{\partial x \partial y^{\prime}}+y^{\prime \prime \prime} \frac{\partial^{2} f}{\partial y^{\prime} \partial y^{\prime \prime}}+y^{1 v} \frac{\partial^{2} f}{\partial y^{\prime} \partial y^{\prime \prime \prime}}+f \frac{\partial^{2} f}{\partial y^{\prime} \partial y^{1 v}} \\
& C=\frac{\partial^{2} f}{\partial x \partial y^{\prime \prime}}+y^{1 v} \frac{\partial^{2} f}{\partial y^{\prime \prime} \partial y^{\prime \prime \prime}}+f \frac{\partial^{2} f}{\partial y^{\prime \prime} \partial y^{1 v}} \\
& D=\frac{\partial^{2} f}{\partial x \partial y^{\prime \prime \prime}}+f \frac{\partial^{2} f}{\partial y^{\prime \prime \prime} \partial y^{1 v}} \\
& E=\frac{\partial^{2} f}{\partial x \partial y^{1 v}} \\
& F=y^{\prime \prime} \frac{\partial f}{\partial y}+y^{\prime \prime \prime} \frac{\partial f}{\partial y^{\prime}}+y^{1 v} \frac{\partial f}{\partial y^{\prime \prime}}+f \frac{\partial f}{\partial y^{\prime \prime \prime}}+f^{\prime} \frac{\partial f}{\partial y^{1 v}} \\
& G=\left(y^{\prime}\right)^{2} \frac{\partial^{2} f}{\partial y^{2}}+\left(y^{\prime \prime}\right)^{2} \frac{\partial^{2} f}{\partial y^{\prime 2}}+\left(y^{\prime \prime \prime}\right)^{2} \frac{\partial^{2} f}{\partial y^{\prime \prime 2}}+\left(y^{1 v}\right)^{2} \frac{\partial^{2} f}{\partial y^{\prime \prime 2}}+f^{2} \frac{\partial^{2} f}{\partial y^{1 v^{2}}} \\
& H=\frac{\partial^{2} f}{\partial x^{2}}
\end{aligned}
$$

\section{RESULTS AND DISCUSSION}

Numerical examples: Two non-linear numerical examples are solved to demonstrate the accuracy and convergence of the new method (10) per iteration for a given value of $x$.

\section{Problem 1:}

$y^{v}=2 y^{\prime} y^{\prime \prime}-y y^{1 v}-y^{\prime} y^{\prime \prime \prime}-8 x+\left(x^{2}-2 x-3\right) e^{x}, 0 \leq x \leq 1, y(0)=$ $1, y^{\prime}(0)=1, y^{\prime \prime}(0)=3, y^{\prime \prime \prime}(0)=1, y^{1 v}(0)=1$.

Theoretical solution is $\mathrm{y}(\mathrm{x})=\mathrm{e}^{\mathrm{x}}+\mathrm{x}^{2}$.

\section{Problem 2:}

$y^{v}=6\left(2\left(y^{\prime}\right)^{3}+6 y y^{\prime} y^{\prime \prime}+y^{2} y^{\prime \prime \prime}\right), 1 \leq x \leq 2, y(1)=1, y^{\prime}(1)=-1$,
$y^{\prime \prime}(1)=2, y^{\prime \prime \prime}(1)=-6, y^{1 v}(1)=24$.

Theoretical solution is $\mathrm{y}(\mathrm{x})=\frac{1}{\mathrm{x}}$. 
Table 1: Solution to Problem 1

\begin{tabular}{llll}
\hline$(\mathrm{n})$ & Exact Sol & method Sol & Error \\
\hline 3 & $0.3936 \mathrm{D}+01$ & $0.3930 \mathrm{D}+01$ & $5.753 \mathrm{D}-03$ \\
5 & $0.3808 \mathrm{D}+01$ & $0.3808 \mathrm{D}+01$ & $8.563 \mathrm{D}-04$ \\
7 & $0.3770 \mathrm{D}+01$ & $0.3770 \mathrm{D}+01$ & $1.996 \mathrm{D}-05$ \\
9 & $0.3753 \mathrm{D}+01$ & $0.3753 \mathrm{D}+01$ & $3.293 \mathrm{D}-06$ \\
11 & $0.3744 \mathrm{D}+01$ & $0.3744 \mathrm{D}+01$ & $1.638 \mathrm{D}-06$ \\
\hline
\end{tabular}

\begin{tabular}{llll}
\multicolumn{2}{l}{ Table 2: Solution to Problem 2 } \\
\hline (n) & Exact Sol & Comp Sol & Error \\
\hline 3 & $0.4915 \mathrm{D}+00$ & $0.4912 \mathrm{D}+00$ & $3.172 \mathrm{D}-04$ \\
5 & $0.4969 \mathrm{D}+00$ & $0.4969 \mathrm{D}+00$ & $1.512 \mathrm{D}-06$ \\
7 & $0.4984 \mathrm{D}+00$ & $0.4984 \mathrm{D}+00$ & $5.303 \mathrm{D}-07$ \\
9 & $0.4990 \mathrm{D}+00$ & $0.4990 \mathrm{D}+00$ & $2.631 \mathrm{D}-07$ \\
11 & $0.4994 \mathrm{D}+00$ & $0.4994 \mathrm{D}+00$ & $2.225 \mathrm{D}-07$ \\
\hline
\end{tabular}

The Table 1 and 2 show the results of Problems 1 and 2 solved with the developed method.

These results Table 1 are recorded at $\mathrm{x}=1$ only for n-iterations.

The results Table 1 and 2 were only recorded for Problems (1) and (2) at $\mathrm{x}=1$ and $\mathrm{x}=2$ respectively, which showed that as the number of iterations (n) are increasing, the new method is converging to the theoretical solutions.

\section{CONCLUSION}

A collocation method with step numbers $\mathrm{k}=5$ has been proposed to solve Problem (1) when $m=5$. Two non-linear test problems were solved by the new method. The results were only recorded for Problems (1) and (2) at $x=1$ and $x=2$ respectively, which showed that as the number of iterations (n) are increasing, the new method is converging to the theoretical solutions.

In our next article for step number $\mathrm{k}=6$ we will compare the accuracy of the present method with that one to ascertain whether an odd or even step number method will be better in terms of accuracy for general purpose use.

\section{REFERENCES}

Adee, S.O., P. Onumanyi, U.W. Sirisena and Y.A. Yahaya, 2005. Note on starting Numerov method more accurately by a hybrid formula of order four for an initial value problem. J. Computat. Applied Math., 175: 369-373. DOI: 10.1016/j.cam.2004.06.016
Awoyemi, D.O., 2003. A P-stable linear multistep method for solving general third order ordinary differential equations. Int. J. Comput. Math., 80: 987-993. DOI: 10.1080/0020716031000079572

Awoyemi, D.O., 2005. Algorithmic collocation approach for direct solution of fourth-order initialvalue problems of ordinary differential equations. Int. J. Comput. Math. 82: 321-329. DOI: 10.1080/00207160412331296634

Awoyemi, D.O. and O.M. Idowu, 2005. A class of hybrid collocation methods for third order ordinary differential equations. Int. J. Comput. Math., 82: 1287-1293. DOI: 10.1080/00207160500112902

Fatunla, S.O., 1988. Numerical Methods for Initial Value Problems in Ordinary Differential Equations. 1st Edn., Academic Press, Inc., New York, ISBN: 0-12-249930-1, pp: 265.

Fatunla, S.O., 1994. A class of block methods for second order IVPs. Int. J. Comput. Math., 55: 119-133. DOI: $10.1080 / 00207169508804368$

Jacques, I. and C.J. Judd, 1987. Numerical Analysis. 1st Edn., Chapman and Hall, New York, ISBN: 0-41227950-9, pp: 233-264.

Kayode, S.J. and D.O. Awoyemi, 2005. A 5-step maximal order method for direct solution of second order ordinary differential equations. J. Nigerian Assoc. Math. Phys., 9: 279-284.

Kayode, S.J., 2008a. An order six zero-stable method for direct solution of fourth-order differential equations. Am. J. Applied Sci., 5: 1461-1466.

Kayode, S.J., 2008b. An efficient zero-stable numerical method for fourth-order differential equations. Int. J. Math. Math. Sci., 2008: 1-10. DOI: $10.1155 / 2008 / 364021$

Lambert, J.D., 1973. Computational Method in Ordinary Differential Equations. 1st Edn., John Willey, New York, ISBN: 0-471-51194-3, pp: 278. 